\title{
A GAIN-OF-FUNCTION MUTATION OF STAT1: A NOVEL GENETIC FACTOR CONTRIBUTING TO CHRONIC MUCOCUTANEOUS CANDIDIASIS
}

\author{
Narges Eslami ${ }^{1,2}$, Marzieh TavaKol ${ }^{3}$, Mehrnaz MesdaGHI ${ }^{2}$, \\ MOHAMMAD GHAREGOZLOU ${ }^{4}$, JEAN-LAURENT CASANOVA ${ }^{5,6,7,8,9}$, \\ ANNE PUEL ${ }^{5,6,7}$, SATOSHI OKADA ${ }^{5,9}$, SABA ARSHI $^{1}$, \\ MOHAMMAD HASSAN BEMANIAN ${ }^{1}$, MORTEZA FALLAHPOUR ${ }^{1}$, \\ RASOOL MOLATEFI ${ }^{1,10}$, FARHAD SEIF ${ }^{11}$, SAMANEH ZOGHI ${ }^{12,13,14}$ \\ NIMA REZAEI ${ }^{12,13,15}$ and MOHAMMAD NABAVI ${ }^{1 *}$ \\ ${ }^{1}$ Department of Allergy and Clinical Immunology, Rasool-e-Akram Hospital, Iran \\ University of Medical Sciences, Tehran, Iran \\ ${ }^{2}$ Department of Allergy and Clinical Immunology, Mofid Children Hospital, Shahid \\ Beheshti University of Medical Sciences, Tehran, Iran \\ ${ }^{3}$ Department of Allergy and Clinical Immunology, Shahid Bahonar Hospital, Alborz \\ University of Medical Sciences, Karaj, Iran \\ ${ }^{4}$ Department of Allergy and Immunology, Children's Medical Center, Tehran University \\ of Medical Sciences, Tehran, Iran \\ ${ }^{5}$ St. Giles Laboratory of Human Genetics of Infectious Diseases, Rockefeller Branch, The \\ Rockefeller University, New York, NY, USA \\ ${ }^{6}$ Laboratory of Human Genetics of Infectious Diseases, Necker Branch, French National \\ Institute of Health and Medical Research (INSERM), Paris, France \\ ${ }^{7}$ Imagine Institute, Paris Descartes University, Paris, France \\ ${ }^{8}$ Pediatric Hematology-Immunology Unit, AP-HP, Necker Hospital for Sick Children, \\ Paris, France \\ ${ }^{9}$ Department of Pediatrics, Hiroshima University Graduate School of Biomedical and \\ Health Sciences, Hiroshima, Japan \\ ${ }^{10}$ Department of Allergy and Clinical Immunology, Bu Ali Children's Hospital, Ardabil \\ University of Medical Sciences, Ardabil, Iran \\ ${ }^{11}$ Department Immunology, School Medicine, Iran University of Medical Sciences, \\ Tehran, Iran \\ ${ }^{12}$ Research Center for Immunodeficiencies, Children's Medical Center, Tehran University \\ of Medical Sciences, Tehran, Iran \\ ${ }^{13}$ Department of Immunology, School of Medicine, Tehran University of Medical \\ Sciences, Tehran, Iran \\ ${ }^{14}$ Network of Immunity in Infection, Malignancy and Autoimmunity (NIIMA), Universal \\ Scientific Education and Research Network (USERN), Vienna, Austria
}

*Corresponding author; E-mail: mnabavi44@yahoo.com 


\footnotetext{
${ }^{15}$ Network of Immunity in Infection, Malignancy and Autoimmunity (NIIMA), Universal Scientific Education and Research Network (USERN), Boston, MA, USA
}

(Received: 9 June 2016; accepted: 22 November 2016)

\begin{abstract}
Heterozygous gain-of-function (GOF) mutations in the signal transducer and activator of transcription 1 (STATI) have increasingly been identified as a genetic cause of autosomal-dominant (AD) chronic mucocutaneous candidiasis (CMC). In this article, we describe a 33-year-old man who experienced chronic refractory candidiasis, recurrent otitis media, and pneumonia resulting in bronchiectasis, severe oral and esophageal candidiases with strictures associated with hypothyroidism and immune hemolytic anemia. His son also suffered from persistent candidiasis, chronic diarrhea, poor weight gain, and pneumonia that resulted in his demise because of sepsis. The immunological workup showed that an inverse CD4/CD8 ratio and serum immunoglobulins were all within normal ranges. The laboratory data revealed failure in response to Candida lymphocyte transformation test. In addition, by Sanger sequencing method, we found a heterozygous mutation, Thr385Met (T385M), located in the DNA-binding domain of STAT1, which was previously shown to be GOF. These findings illustrate the broad and variable clinical phenotype of heterozygous STAT1 GOF mutations. However, more clinical information and phenotype-genotype studies are required to define the clinical phenotype caused by AD STAT1 GOF.
\end{abstract}

Keywords: gain-of-function mutations in STAT1, chronic mucocutaneous candidiasis

\title{
Introduction
}

Chronic mucocutaneous candidiasis (CMC) is characterized by persistent or recurrent Candida infections of the skin, nails, and mucosal membranes, which may poorly respond to antifungal treatment or relapse upon discontinuation of treatment [1]. It is often associated with a variety of infectious diseases as well as autoimmune or endocrine disorders such as hypothyroidism [2]. CMC is frequent in immunodeficiencies affecting $\mathrm{T}$ cell number and/or function, associated with various infectious and/or autoimmune manifestations $[1,2]$. Patients with syndromic CMC and primary immunodeficiencies have been displaying impaired interleukin (IL) 17 immunity [1]. IL-17-mediated immunity has recently been recognized as crucial in the human mucocutaneous defense against Candida [1-6]. The mutations selectively abolishing IL-17-mediated immunity are linked to pathogenesis of CMC [4-6]. CMC is a prominent feature of autosomal-dominant (AD) hyper immunoglobulin E (IgE) syndrome [7, 8], autosomal-recessive (AR) autoimmune polyendocrinopathy candidiasis with ectodermal dystrophy syndrome [9-11], 
AR Caspase Recruitment Domain-containing protein 9 deficiency with invasive fungal infection [12,13], and Mendelian susceptibility to mycobacterial disease (MSMD) with AR IL-12p40 or IL-12R $\beta 1$ deficiency $[7,14,15]$. CMC can also be found as one of the main clinical presentations or the only clinical presentation in patients without any of the previously mentioned underlying causes of CMC disease (CMCD) $[16,17]$. AD heterozygous gain-of-function (GOF) mutations in the signal transducer and activator of transcription 1 (STAT1) are the most common genetic etiology of CMC disease which impaires the development of IL-17-producing T cells [18-24]. STAT1 mutations cause a wide spectrum of diseases, ranging from severe intracellular bacterial and viral infections (biallelic loss-of-function mutations) to MSMD (monoallelic loss-of-function mutations) and CMC with other infectious and/or autoimmune manifestations (monoallelic GOF mutations) [18, 22, 25]. Most of these mutations affect the coiled-coil domain or DNA-binding domain (DBD) of STAT1. They increase STAT1 phosphorylation and cellular responses to STAT1-dependant cytokines, such as interferon (IFN)- $\alpha / \beta$, IFN- $\gamma$, IL-27, and STAT3-dependent IL-6 and IL-21 [5, 18, 26]. In parallel, they inhibit the development of IL-17-producing T cells [5, 18, 26, 27]. Autoimmunity probably results from stronger IFN- $\alpha / \beta$ signaling as it is a frequent adverse effect of treatment with recombinant IFN- $\alpha$ or IFN- $\beta$. In addition, some autoimmunity signs in patients treated with IFN- $\alpha$ (e.g., thyroiditis) [20, 28].

\section{Case Report}

A 33-year-old male has been referred to our clinic with a history of recurrent oral thrush and productive cough soon after the birth. He was the eighth child of unrelated parents with seven healthy siblings. He had a history of chronic refractory candidiasis affecting different sites of his skin, mucous membranes, and nails since early childhood. He was diagnosed with hiatal hernia with remarkable gastroesophageal reflux at age of 11 months that required a surgical intervention. He also suffered from recurrent otitis media and otorrhea since early infancy that led to tympanomastoidectomy at the age of 12 years. Frequent episodes of pneumonia since his childhood resulted in multiple hospitalizations with development of clubbing, focal bronchiectasis, and partial pulmonary fibrosis (Figure 1). Prophylactic oral itraconazole administered with initial diagnosis of $\mathrm{CMC}$ at the age of 14 years resulted in partial control of oral candidiasis. He also suffered from recurrent aphthous stomatitis, chronic diarrhea, growth retardation, and delayed puberty. He was diagnosed with hypothyroidism at the age of 18 years and treated with thyroid hormone replacement. At the age of 21 years, he developed severe esophageal candidiasis with resultant strictures associated with dysphagia and 


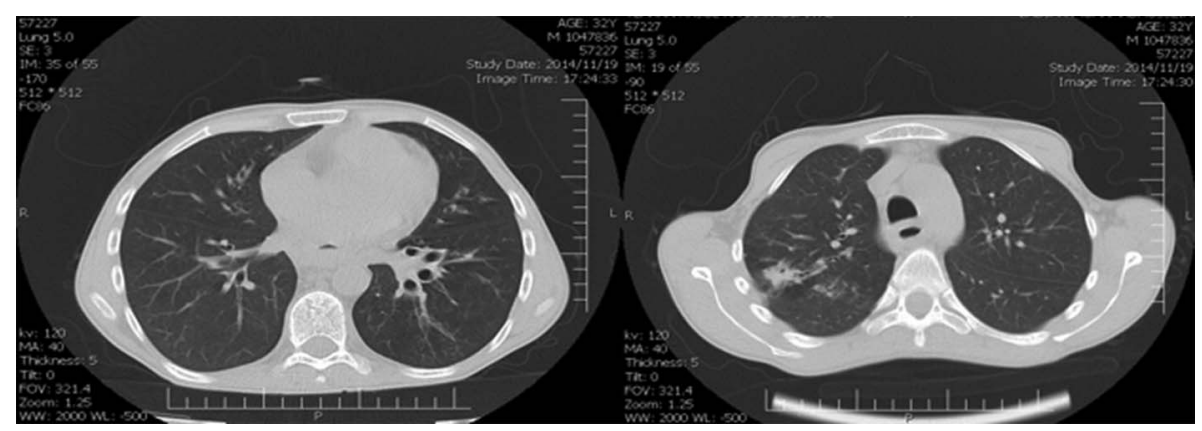

Figure 1. Chest CT scan of the patient with GOF mutation of STAT1 focal bronchiectasis and partial pulmonary fibrosis

odynophagia that led to multiple esophageal dilatations. When he was 27 years old, he experienced multiple episodes of acute immune hemolytic anemia with warm autoantibodies with positive Coombs' test (Table I). High doses of prednisolone were not tolerated by the patient; hence intravenous immunoglobulins were begun and advised to be injected every month. His son also suffered from persistent whitish oral plaques, chronic diarrhea, poor weight gain, and pneumonia since birth, which resulted in his demise because of the sepsis at the sixth month of life. Azole-resistant Candida albicans was isolated from his oral lesions.

\section{Results}

Flow cytometry revealed an inverse CD4/CD8 ratio and his serum immunoglobulins were all within normal ranges (Table I). The laboratory data showed impaired lymphocyte proliferative response to Candida and Bacillus Calmette-Guérin antigens [impaired lymphocyte transformation test (LTT)] (Table I). Sputum analysis and culture for acid-fast bacilli were negative. In addition, on genetic study, we found that both of the patient and his son had a heterozygous DBD GOF mutation at Thr385Met (T385M) in the STAT1 gene [29] (Figures 2 and 3).

\section{Discussion}

According to previous reports, patients with STAT1 GOF mutations are expected to have a broader immunological and infectious phenotype, given the role of STAT1 in multiple signaling pathways [18]. Several patients with STAT1 GOF mutations possess not only $\mathrm{CMC}$, but also severe viral and mycobacterial 
Table I. Laboratory results of the patient with CMC

\begin{tabular}{|c|c|c|c|c|}
\hline Variable & Amount & Unit & Normal range & Comment \\
\hline \multicolumn{5}{|l|}{ Immunoglobulin levels } \\
\hline $\operatorname{IgG}$ & 1,022 & $\mathrm{mg} / \mathrm{dl}$ & $767-1,590$ & Normal \\
\hline IgA & 346 & $\mathrm{mg} / \mathrm{dl}$ & $61-356$ & Normal \\
\hline IgM & 159 & $\mathrm{mg} / \mathrm{dl}$ & $37-286$ & Normal \\
\hline $\operatorname{IgE}$ & 20 & $\mathrm{IU} / \mathrm{ml}$ & $<100$ & Normal \\
\hline \multicolumn{5}{|l|}{ Flow cytometry } \\
\hline $\mathrm{CD} 3+\mathrm{T}$ cell & 66 & $\%$ & $55-82$ & Normal \\
\hline $\mathrm{CD} 4+\mathrm{T}$ cell & 26 & $\%$ & $25-75$ & Lower limit normal \\
\hline $\mathrm{CD} 8+\mathrm{T}$ cell & 41 & $\%$ & $14-34$ & High \\
\hline CD16+ NK cell & 2.6 & $\%$ & $6-31$ & Low \\
\hline CD56+ NK cell & 6 & $\%$ & $6-31$ & Normal \\
\hline CD19+ B cell & 20 & $\%$ & $6-23$ & Normal \\
\hline CD20+ B cell & 19 & $\%$ & $6-23$ & Normal \\
\hline \multicolumn{5}{|l|}{ Others } \\
\hline NBT & 98 & $\%$ & $>90 \%$ & Normal \\
\hline WBC & $5.3 \times 10^{3}$ & cells $/ \mu 1$ & $4.5-13.5 \times 10^{3}$ & Normal \\
\hline $\mathrm{Hb}$ & 6.3 & $\mathrm{~g} / \mathrm{dl}$ & $12-15$ & Normal \\
\hline PLT & $199 \times 10^{3}$ & cells $/ \mu 1$ & $140,000-440,000$ & Normal \\
\hline Corrected retic & 6.7 & $\%$ & $0.5-2.5$ & High \\
\hline Neutrophil & 62 & $\%$ & & Normal \\
\hline Lymphocyte & 32 & $\%$ & & Normal \\
\hline Monocyte & 6 & $\%$ & & Normal \\
\hline Coombs' test/indirect & Positive & & Negative & \\
\hline Anti-IgG, -C3d & Positive & & & \\
\hline Anti-IgG & Positive $(+3)$ & & & \\
\hline Anti-C3d & Positive $(+4)$ & & & \\
\hline $\mathrm{C} 3$ & 84 & $\mathrm{mg} / \mathrm{dl}$ & $90-180$ & \\
\hline $\mathrm{C} 4$ & 40 & $\mathrm{mg} / \mathrm{dl}$ & $10-40$ & \\
\hline $\mathrm{CH} 50$ & 94 & $\%$ & $>90 \%$ & \\
\hline LTT BCG & $<0.75$ & RPI & $0.75-1.65$ & Low \\
\hline LTT Candida & $<0.75$ & RPI & $0.75-1.65$ & Low \\
\hline LTT PHA & 0.85 & RPI & $0.75-1.65$ & Normal \\
\hline
\end{tabular}

Note: NBT: nitro blue tetrazolium; WBC: white blood cell; Hb: hemoglobin; PLT: platelet; RPI: Railway Performance Index; CH50: total hemolytic complement; PHA: phytohemagglutinin; LTT: lymphocyte transformation test.

infections and/or autoimmune diseases [18, 21, 24]. However, our case did not have any problems with severe viral or mycobacterial infection. Takezaki et al. [19] and Sharfe et al. [23] reported patients with the same T385M GOF STAT1 allele who had $\mathrm{CMC}$, recurrent lower respiratory tract infections, bronchiectasis, and autoimmunities similar to the case described here. Moreover, this case had diaphragmatic hernia and esophageal dysmotility which seems to be a noteworthy feature of this syndrome as hypothesized by Uzel et al. [22] and Frans et al. [30]. 


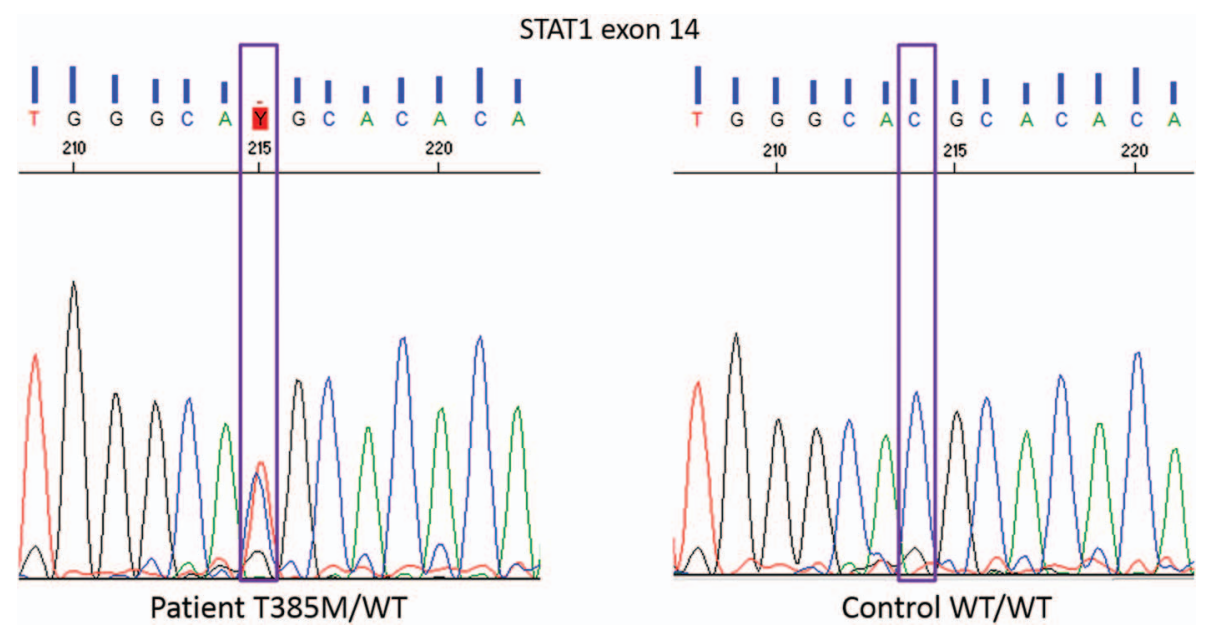

Figure 2. Direct sequence analysis of STAT1 exon 14 in patient revealed base change of c. $1153 \mathrm{C}>\mathrm{T}$ resulting in p.T385M in STAT1

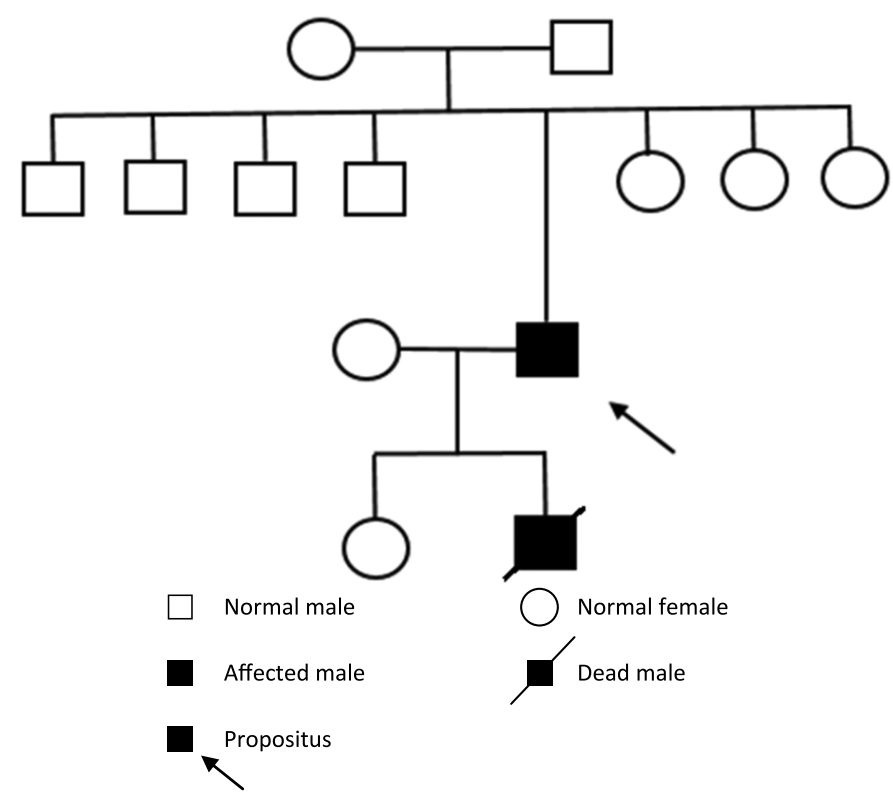

Figure 3. The pedigree of the family

Whether the gastrointestinal manifestations are all secondary to CMC or a primary manifestation of disturbed STAT1 signaling has yet to be determined [20, 22]. Soltész et al. [20] and Depner et al. [31] described patients carrying GOF 
mutations of STAT1 with developed multiple intracranial aneurysms, but our patient fortunately had no cerebral vascular complications.

Increased susceptibility to Fas activation in $T 385 M \mathrm{~T}$ cells induces cell apoptosis which might contribute to the progressive decline in $\mathrm{T}$ cell numbers [23]. These findings show that some heterozygous aberrations of STAT1 can be associated with progressive combined immunodeficiency, accompanied by lifethreatening severe infections. These infections increase with age, sometimes into their second decade of life, accompanied by variable autoimmune features [23]. This finding suggests that these STATI GOF mutations may ultimately be fatal due to overwhelming infections $[19,23,32,33]$. However, we discuss here a patient with a relatively long life span.

These data suggest that heterozygous STATI GOF mutations underlie an unexpectedly broad clinical phenotype. More clinical information and phenotypegenotype studies are required to determine the clinical phenotype caused by AD GOF mutations of STATI.

\section{Conflict of Interest}

The authors also declare that there is no conflict of interest.

\section{References}

1. Puel, A., Picard, C., Cypowyj, S., Lilic, D., Abel, L., Casanova, J. L.: Inborn errors of mucocutaneous immunity to Candida albicans in humans: A role for IL-17 cytokines? Curr Opin Immunol 22, 467-474 (2010).

2. Puel, A., Cypowyj, S., Marodi, L., Abel, L., Picard, C., Casanova, J. L.: Inborn errors of human IL-17 immunity underlie chronic mucocutaneous candidiasis. Curr Opin Allergy Clin Immunol 12, 616-622 (2012).

3. Picard, C., Maródi, L., Casanova, J. L., Puel, A.: Immunity to infection in IL-17-deficient mice and humans. Eur J Immunol 42, 2246-2254 (2012).

4. Ling, Y., Puel, A.: IL-17 and infections. Actas Dermosifiliogr 105, 34-40 (2014).

5. Lanternier, F., Cypowyj, S., Picard, C., Bustamante, J., Lortholary, O., Casanova, J. L., Puel, A.: Primary immunodeficiencies underlying fungal infections. Curr Opin Pediatr 25, 736-747 (2013).

6. Ling, Y., Cypowyj, S., Aytekin, C., Galicchio, M., Camcioglu, Y., Nepesov, S., Ikinciogullari, A., Dogu, F., Belkadi, A., Levy, R., Migaud, M., Boisson, B., Bolze, A., Itan, Y., Goudin, N., Cottineau, J., Picard, C., Abe, L. L., Bustamante, J., Casanova, J. L., Puel, A.: Inherited IL-17RC deficiency in patients with chronic mucocutaneous candidiasis. J Exp Med 212, 619-631 (2015).

7. de Beaucoudrey, L., Puel, A., Filipe-Santos, O., Cobat, A., Ghandil, P., Chrabieh, M., Feinberg, J., von Bernuth, H., Samarina, A., Jannière, L., Fieschi, C., Stéphan, J. L., 
Boileau, C., Lyonnet, S., Jondeau, G., Cormier-Daire, V., Le Merrer, M., Hoarau, C., Lebranchu, Y., Lortholary, O., Chandesris, M. O., Tron, F., Gambineri, E., Bianchi, L., Rodriguez-Gallego, C., Zitnik, S. E., Vasconcelos, J., Guedes, M., Vitor, A. B., Marodi, L., Chapel, H., Reid, B., Roifman, C., Nadal, D., Reichenbach, J., Caragol, I., Garty, B. Z., Dogu, F., Camcioglu, Y., Gülle, S., Sanal, O., Fischer, A., Abel, L., Stockinger, B., Picard, C., Casanova, J. L.: Mutations in STAT3 and IL12RBI impair the development of human IL-17-producing T cells. J Exp Med 205, 1543-1550 (2008).

8. Renner, E. D., Rylaarsdam, S., Anover-Sombke, S., Rack, A. L., Reichenbach, J., Carey, J. C., Zhu, Q., Jansson, A. F., Barboza, J., Schimke, L. F., Leppert, M. F., Getz, M. M., Seger, R. A., Hill, H. R., Belohradsky, B. H., Torgerson, T. R., Ochs, H. D.: Novel signal transducer and activator of transcription 3 (STAT3) mutations, reduced TH17 cell numbers, and variably defective STAT3 phosphorylation in hyper-IgE syndrome. J Allergy Clin Immunol 122, 181-187 (2008).

9. Puel, A., Döffinger, R., Natividad, A., Chrabieh, M., Barcenas-Morales, G., Picard, C., Cobat, A., Ouachée-Chardin, M., Toulon, A., Bustamante, J., Al-Muhsen, S., Al-Owain, M., Arkwright, P. D., Costigan, C., McConnell, V., Cant, A. J., Abinun, M., Polak, M., Bougnères, P. F., Kumararatne, D., Marodi, L., Nahum, A., Roifman, C., Blanche, S., Fischer, A., Bodemer, C., Abel, L., Lilic, D., Casanova, J. L.: Autoantibodies against IL-17A, IL7F, and IL-22 in patients with chronic mucocutaneous candidiasis and autoimmune polyendocrine syndrome type I. J Exp Med 207, 291-297 (2010).

10. Kisand, K., Bøe Wolff, A. S., Podkrajsek, K. T., Tserel, L., Link, M., Kisand, K. V., Ersvaer, E., Perheentupa, J., Erichsen, M. M., Bratanic, N., Meloni, A., Cetani, F., Perniola, R., Ergun-Longmire, B., Maclaren, N., Krohn, K. J., Pura, M., Schalke, B., Ströbel, P., Leite, M. I., Battelino, T., Husebye, E. S., Peterson, P., Willcox, N., Meager, A.: Chronic mucocutaneous candidiasis in APECED or thymoma patients correlates with autoimmunity to Th17-associated cytokines. J Exp Med 207, 299-308 (2010).

11. Kisand, K., Peterson, P.: Autoimmune polyendocrinopathy candidiasis ectodermal dystrophy: Known and novel aspects of the syndrome. Ann N Y Acad Sci 1246, 77-91 (2011).

12. Glocker, E. O., Hennigs, A., Nabavi, M., Schaffer, A. A., Woellner, C., Salzer, U., Pfeifer, D., Veelken, H., Warnatz, K., Tahami, F., Jamal, S., Manguiat, A., Rezaei, N., Amirzargar, A. A., Plebani, A., Hannesschlager, N., Gross, O., Ruland, J., Grimbacher, B.: A homozygous CARD9 mutation in a family with susceptibility to fungal infections. N Engl J Med 361, 1727-1735 (2009).

13. Lanternier, F., Mahdaviani, S. A., Barbati, E., Chaussade, H., Koumar, Y., Levy, R., Denis, B., Brunel, A. S., Martin, S., Loop, M., Peeters, J., de Selys, A., Vanclaire, J., Vermylen, C., Nassogne, M. C., Chatzis, O., Liu, L., Migaud, M., Pedergnana, V., Desoubeaux, G., Jouvion, G., Chretien, F., Darazam, I. A., Schäffer, A. A., Netea, M. G., De Bruycker, J. J., Bernard, L., Reynes, J., Amazrine, N., Abel, L., Van der Linden, D., Harrison, T., Picard, C., Lortholary, O., Mansouri, D., Casanova, J. L., Puel, A.: Inherited CARD9 deficiency in otherwise healthy children and adults with Candida species-induced meningoencephalitis, colitis, or both. J Allergy Clin Immunol 135, 1558-1568 (2015).

14. Ouederni, M., Sanal, O., Ikinciogullari, A., Tezcan, I., Dogu, F., Sologuren, I., PedrazaSánchez, S., Keser, M., Tanir, G., Nieuwhof, C., Colino, E., Kumararatne, D., Levy, J., Kutukculer, N., Aytekin, C., Herrera-Ramos, E., Bhatti, M., Karaca, N., Barbouche, R., Broides, A., Goudouris, E., Franco, J. L., Parvaneh, N., Reisli, I., Strickler, A., Shcherbina, A., Somer, A., Segal, A., Angel-Moreno, A., Lezana-Fernandez, J. L., Bejaoui, M., Bobadilla-Del 
Valle, M., Kachboura, S., Sentongo, T., Ben-Mustapha, I., Bustamante, J., Picard, C., Puel, A., Boisson-Dupuis, S., Abel, L., Casanova, J. L., Rodríguez-Gallego, C.: Clinical features of candidiasis in patients with inherited interleukin 12 receptor $\beta 1$ deficiency. Clin Infect Dis $\mathbf{5 8}$, 204-213 (2014).

15. Prando, C., Samarina, A., Bustamante, J., Boisson-Dupuis, S., Cobat, A., Picard, C., AlSum, Z., Al-Jumaah, S., Al-Hajjar, S., Frayha, H., Alangari, A., Al-Mousa, H., Mobaireek, K. F., Ben-Mustapha, I., Adimi, P., Feinberg, J., de Suremain, M., Jannière, L., Filipe-Santos, O., Mansouri, N., Stephan, J. L., Nallusamy, R., Kumararatne, D. S., Bloorsaz, M. R., Ben-Ali, M., Elloumi-Zghal, H., Chemli, J., Bouguila, J., Bejaoui, M., Alaki, E., AlFawaz, T. S., Al Idrissi, E., ElGhazali, G., Pollard, A. J., Murugasu, B., Wah Lee, B., Halwani, R., Al-Zahrani, M., Al Shehri, M. A., Al-Zahrani, M., Bin-Hussain, I., Mahdaviani, S. A., Parvaneh, N., Abel, L., Mansouri, D., Barbouche, R., Al-Muhsen, S., Casanova, J. L.: Inherited IL-12p40 deficiency: Genetic, immunologic, and clinical features of 49 patients from 30 kindreds. Medicine (Baltimore) 92, 109-122 (2013).

16. Puel, A., Cypowyj, S., Bustamante, J., Wright, J. F., Liu, L., Lim, H. K., Migaud, M., Israel, L., Chrabieh, M., Audry, M., Gumbleton, M., Toulon, A., Bodemer, C., El-Baghdadi, J., Whitters, M., Paradis, T., Brooks, J., Collins, M., Wolfman, N. M., Al-Muhsen, S., Galicchio, M., Abel, L., Picard, C., Casanova, J. L.: Chronic mucocutaneous candidiasis in humans with inborn errors of interleukin-17 immunity. Science 332, 65-68 (2011).

17. Casanova, J. L., Abel, L.: The genetic theory of infectious diseases: A brief history and selected illustrations. Annu Rev Genomics Hum Genet 14, 215-243 (2013).

18. Liu, L., Okada, S., Kong, X. F., Kreins, A. Y., Cypowyj, S., Abhyankar, A., Toubiana, J., Itan, Y., Audry, M., Nitschke, P., Masson, C., Toth, B., Flatot, J., Migaud, M., Chrabieh, M., Kochetkov, T., Bolze, A., Borghesi, A., Toulon, A., Hiller, J., Eyerich, S., Eyerich, K., Gulácsy, V., Chernyshova, L., Chernyshov, V., Bondarenko, A., Grimaldo, R. M., Blancas-Galicia, L., Beas, I. M., Roesler, J., Magdorf, K., Engelhard, D., Thumerelle, C., Burgel, P. R., Hoernes, M., Drexel, B., Seger, R., Kusuma, T., Jansson, A. F., SawalleBelohradsky, J., Belohradsky, B., Jouanguy, E., Bustamante, J., Bué, M., Karin, N., Wildbaum, G., Bodemer, C., Lortholary, O., Fischer, A., Blanche, S., Al-Muhsen, S., Reichenbach, J., Kobayashi, M., Rosales, F. E., Lozano, C. T., Kilic, S. S., Oleastro, M., Etzioni, A., Traidl-Hoffmann, C., Renner, E. D., Abel, L., Picard, C., Maródi, L., BoissonDupuis, S., Puel, A., Casanova, J. L.: Gain-of-function human STAT1 mutations impair IL-17 immunity and underlie chronic mucocutaneous candidiasis. J Exp Med 208, 1635-1648 (2011).

19. Takezaki, S., Yamada, M., Kato, M., Park, M. J. Maruyama, K., Yamazaki, Y., Chida, N., Ohara, O., Kobayashi, I., Ariga, T.: Chronic mucocutaneous candidiasis caused by a gainof-function mutation in the STAT1 DNA-binding domain. J Immunol 189, 1521-1526 (2012).

20. Soltész, B., Tóth, B., Shabashova, N., Bondarenko, A., Okada, S., Cypowyj, S., Abhyankar, A., Csorba, G., Taskó, S., Sarkadi, A. K., Méhes, L., Rozsíval, P., Neumann, D., Chernyshova, L., Tulassay, Z., Puel, A., Casanova, J. L., Sediva, A., Litzman, J., Maródi, L.: New and recurrent gain-of-function STAT1 mutations in patients with chronic mucocutaneous candidiasis from Eastern and Central Europe. J Med Genet 50, 567-578 (2013).

21. van de Veerdonk, F. L., Plantinga, T. S., Hoischen, A., Smeekens, S. P., Joosten, L. A., Gilissen, C., Arts, P., Rosentul, D. C., Carmichael, A. J., Smits-van der Graaf, C. A., Kullberg, B. J., van der Meer, J. W., Lilic, D., Veltman, J. A., Netea, M. G.: STAT1 
mutations in autosomal dominant chronic mucocutaneous candidiasis. N Engl J Med 365, 54-61 (2011).

22. Uzel, G., Sampaio, E. P., Lawrence, M. G., Hsu, A. P., Hackett, M., Dorsey, M. J., Noel, R. J., Verbsky, J. W., Freeman, A. F., Janssen, E., Bonilla, F. A., Pechacek, J., Chandrasekaran, P., Browne, S. K., Agharahimi, A., Gharib, A. M., Mannurita, S. C., Yim, J. J., Gambineri, E., Torgerson, T., Tran, D. Q., Milner, J. D., Holland, S. M.: Dominant gain-of- function STAT1 mutations in FOXP3 wild-type immune dysregulationpolyendocrinopathy-enteropathy-X-linked-like syndrome. J Allergy Clin Immunol 131, 1611-1623 (2013).

23. Sharfe, N., Nahum, A., Newell, A., Dadi, H., Ngan, B. L., Pereira, S., Herbrick, J., Rofiman, C.: Fatal combined immunodeficiency associated with heterozygous mutation in STAT1. J Allergy Clin Immunol 133, 807-817 (2014).

24. Tóth, B., Méhes, L., Taskó, S., Szalai, Z., Tulassay, Z., Cypowyj, S., Casanova, J. L., Puel, A., Maródi, L.: Herpes in gain-of-function STAT1 mutation. Lancet 379, 2500-2561 (2012).

25. Boisson-Dupuis, S., Kong, X. F., Okada, S., Cypowyj, S., Puel, A., Abel, L., Casanova, J. L.: Inborn errors of human STAT1: Allelic heterogeneity governs the diversity of immunological and infectious phenotypes. Curr Opin Immunol 24, 364-378 (2012).

26. Boisson, B., Quartier, P., Casanova, J. L.: Immunological loss-of-function due to genetic gain-of-function in humans: Autosomal dominance of the third kind. Curr Opin Immunol 32, 90-105 (2015).

27. Ramgolam, V. S., Sha, Y., Jin, J., Zhang, X., Markovic-Plese, S.: IFN- $\beta$ inhibits human Th17 cell differentiation. J Immunol 183, 5418-5427 (2009).

28. Selmi, C., Lleo, A., Zuin, M., Podda, M., Rossaro, L., Gershwin, M. E.: Interferon $\alpha$ and its contribution to autoimmunity. Curr Opin Investig Drugs 7, 451-456 (2006).

29. Toubiana, J., Okada, S., Hiller, J., Oleastro, M., Lagos Gomez, M., Aldave Becerra, J. C., Ouachée-Chardin, M., Fouyssac, F., Girisha, K. M., Etzioni, A., Van Montfrans, J., Camcioglu, Y., Kerns, L. A., Belohradsky, B., Blanche, S., Bousfiha, A., RodriguezGallego, C., Meyts, I., Kisand, K., Reichenbach, J., Renner, E. D., Rosenzweig, S., Grimbacher, B., van de Veerdonk, F. L., Traidl-Hoffmann, C., Picard, C., Marodi, L., Morio, T., Kobayashi, M., Lilic, D., Milner, J. D., Holland, S., Casanova, J. L., Puel, A.: International STAT1 Gain-of-Function Study Group: Heterozygous STAT1 gain-offunction mutations underlie an unexpectedly broad clinical phenotype. Blood 127, 3154-3164 (2016).

30. Frans, G., Moens, L., Schaballie, H., Van Eyck, L., Borgers, H., Wuyts, M., Dillaerts, D., Vermeulen, E., Grimbacher, B., Cant, A., Declerck, D., Peumans, M., Renard, M., De Boeck, K., Hoffman, I., François, I., Liston, A., Claessens, F., Bossuyt, X., Meyts, I.: Gain-of-function mutations in signal transducer and activator of transcription 1 (STAT1): Chronic mucocutaneous candidiasis accompanied by enamel defects and delayed dental shedding. J Allergy Clin Immunol 134, 1209-1213 (2014).

31. Depner, M., Fuchs, S., Raabe, J., Frede, N., Glocker, C., Doffinger, R., Gkrania-Klotsas, E., Kumararatne, D., Atkinson, T. P., Schroeder, H. W., Jr., Niehues, T., Dückers, G., StrayPedersen, A., Baumann, U., Schmidt, R., Franco, J. L., Orrego, J., Ben-Shoshan, M., McCusker, C., Abe Jacob, C. M., Carneiro-Sampaio, M., Devlin, L. A., Edgar, J. D. M., Henderson, P., Russell, R. K., Skytte, A.-B., Seneviratne, S. L., Wanders, J., Stauss, H., Meyts, I., Moens, L., Jesenak, M., Kobbe, R., Borte, S., Borte, M., Wright, D. A., Hagin, 
D., Torgerson, T. R., Grimbacher, B.: The extended clinical phenotype of 26 patients with chronic mucocutaneous candidiasis due to gain-of-function mutations in STAT1. J Clin Immunol 36, 73-84 (2016).

32. Ruda Wessell, K. M., Tcheurekdjian, H., Hostoffer, R.: Autosomal dominant transmission of signal transduction and activator of transcription 1 (STAT 1) mutation (Thr385Met) and extended lifespan. LymphoSign J 3, 13-17 (2016).

33. Roifman, C. M: Monoallelic STAT1 mutations and disease patterns. LymphoSign J 1, 57-59 (2014). 